\title{
RELATIONSHIP BETWEEN FRACTIONAL SEPARATION CURVES AND QUANTITATIVE OPTIMIZATION CRITERIA IN THE SEPARATION OF POURABLE MATERIALS
}

\author{
E. BARSKY $Y^{\mathrm{a}}$ and M. BARSKY ${ }^{\mathrm{b}, *}$ \\ ${ }^{a}$ Negev Academic College of Engineering, Beer-Sheva, Israel; \\ ${ }^{\mathrm{b}}$ The Institutes for Applied Research, Ben-Gurion University of the Negev, P.O.B. 653, \\ Beer-Sheva 84105, Israel
}

(Received 6 January 2004; In final form 24 February 2004)

\begin{abstract}
For more than 60 years, the accepted wisdom has been that the methods of optimizing separation processes based on quantitative criteria are fundamentally different from those based on separation curves. The present article demonstrates a functional relation between these two approaches. Reposing upon this relation, we propose a new method for the optimization of separation processes, which combines the advantages of both approaches, namely the availability of a quantitative evaluation and invariance of the evaluation with respect to the grain-size distribution of the starting material.
\end{abstract}

Keywords: Separation curve; Particle size; Granulometric composition; Density

\section{INTRODUCTION}

Regardless of the technology used, the purpose of a separator is always the same: to partition the original material into two products along a certain size boundary so as to obtain the greatest possible extent of separation. In any separation, the maximization of difference in grain-size distribution among the end products requires that the operation be carried out under an optimal regime. In order to identify conditions that provide the optimal efficacy, let us examine, in its general form, the redistribution of particulate fractions that takes place in the course of separation.

*Corresponding author. E-mail: barskym@bgumail.bgu.ac.il 


\section{QUANTITATIVE CRITERIONS OF OPTIMIZATION}

Let us suppose that a given pourable material has the initial grain-size distribution characterized by the curve ABC in Fig. 1. Let us further assume that, for technical reasons, this material has to be separated along a certain grain-size boundary that is denoted by $x_{0}$. On the given scale, the area on the graph bounded by the curve ABC and the coordinate axes represents the total amount of material introduced into the classifier. We take this amount to be equal to unity and denote the curve expressing the initial distribution by $Q(x)$. In the ideal case, the starting material should become separated along the $B x_{0}$ line. With reference to that line, the feed consists of two parts: fine $\left(D_{s}\right)$ and coarse $\left(R_{S}\right)$.

In the real processes, separation does not proceed in the ideal manner, because a portion of the fine fractions finds its way into the coarse product and a portion of the coarse fractions into the fine product. The fine product is then described by the curve $q(x)$ and the coarse product by $n(x)$. From the way in which these curves were derived, it is obvious that at any point $x_{i}$ the following relation is satisfied:

$$
Q\left(x_{1}\right)=q\left(x_{i}\right)+n\left(x_{i}\right)
$$

The degree of recovery of the given granulate fraction is given by:

$$
F_{f}\left(x_{1}\right)+F_{c}\left(x_{i}\right)=1
$$

where $F_{f}\left(x_{i}\right)=q\left(x_{i}\right) / Q\left(x_{i}\right)$ is the yield of the particulate fraction into the fine product and $F_{c}\left(x_{i}\right)=n\left(x_{i}\right) / Q\left(x_{i}\right)$ is the yield of the particulate fraction into the coarse product.

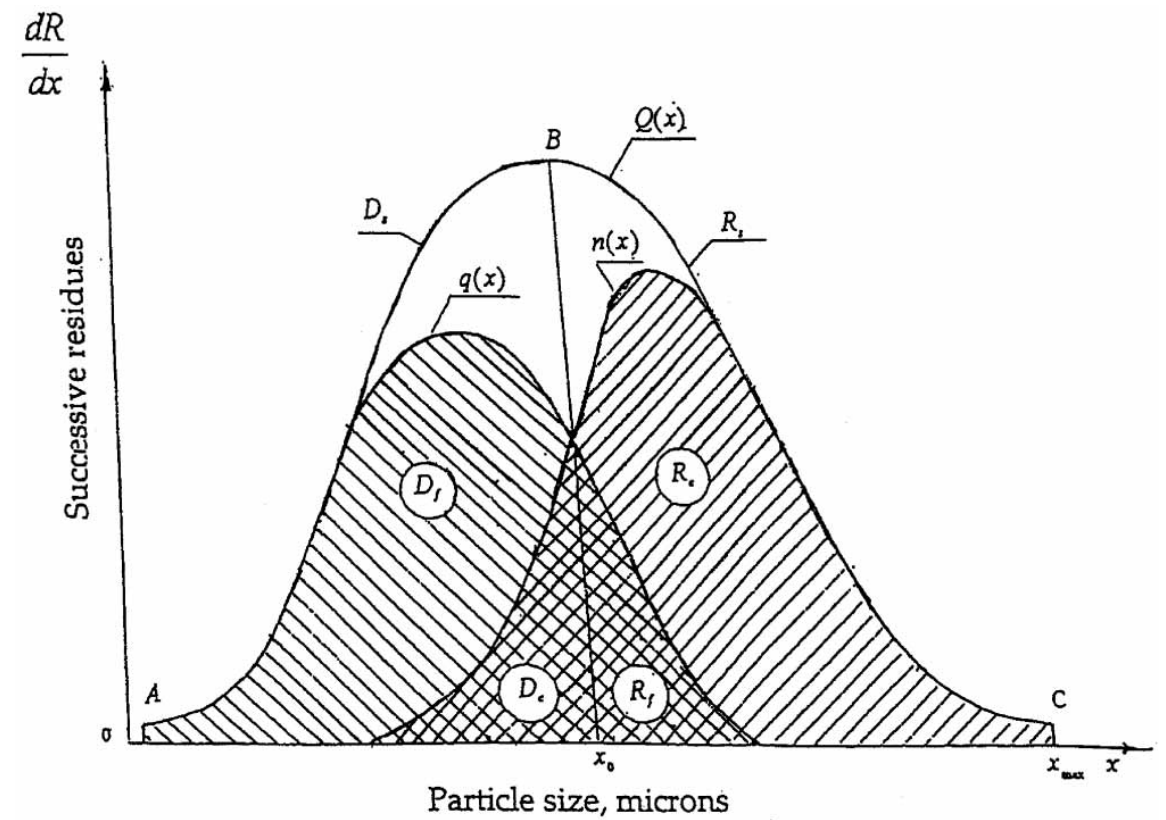

FIGURE 1 Grain-size distribution of the successive residues in the separation of a pourable material. 
Figure 2 presents the separation curves, obtained using Fig. 1, for the fine and coarse products in the form:

$$
F_{f}(x)=f(x) ; \quad F_{c}(x)=f(x)
$$

The area of the graph in Fig. 1 is divided into four zones by the lines of the ideal and the real processes: $D_{f}$ - fine material at the outlet of the fine product; $R_{f}$-coarse material at the outlet of the fine product; $R_{c}$-coarse material at the outlet of the coarse product; $D_{c}$-fine material at the outlet of the coarse product.

The following relations hold for these zones:

$$
\begin{array}{ll}
D_{s}+R_{s}=1 ; & R_{f}+D_{f}=\gamma_{f} \\
D_{f}+D_{c}=D_{s} ; & R_{c}+D_{c}=\gamma \\
R_{c}+R_{f}=R_{s} ; & \gamma_{f}+\gamma_{c}=1
\end{array}
$$

where $\gamma_{f}$ and $\gamma_{c}$ are the relative yields of fine and coarse products, respectively.

In the current theory and practice of optimization of separation processes, two different approaches have emerged: one based on quantitative quality criteria and the other on separation curves.

The first approach is associated with the name of Hancock [1,2]. This author proposed an objective criterion consisting of relations expressing the output of the fine product:

$$
E_{f}=\frac{D_{f}}{D}-\frac{R_{f}}{R_{s}}
$$

and coarse product:

$$
E_{c}=\frac{R_{c}}{R_{s}}-\frac{D_{c}}{D_{s}}
$$

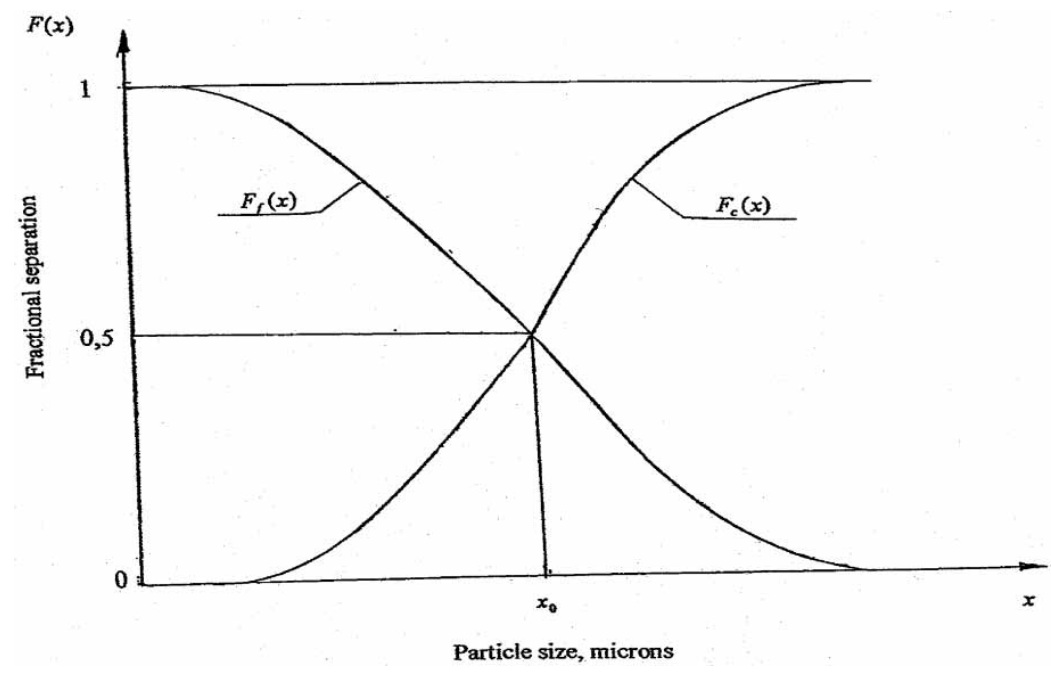

FIGURE 2 Fractional separation curves. 
The advantage of this method is that it provides a numerical criterion for the quality of separation. This feature makes it possible to optimize the process, as well as to compare the maximum efficiencies of different classifiers. The criterion is monotonous, i.e. the greater the fractional difference between the separation products, the larger the numerical value of the criterion. For an ideal separation, where $D_{f}=D_{s}$ and $R_{c}=R_{s}$, the criterion gives the maximum grade of $E=1$. When the starting material is divided into products without any change taking place in its fractional composition, that is for $D_{f}=m D_{s}$ and $R_{f}=m R_{s}$, the grade is zero, i.e. $E=0$. Thus the behaviour of the criterion reflects the meaning of the process of separation.

In addition, the efficacy determined according to these criteria is always the same independent of the product used to calculate its value. In order to prove this, it is sufficient to subtract one formula from the other in Eq. (4):

$$
E_{f}-E_{c}=\frac{D_{f}}{D_{s}}-\frac{R_{f}}{R_{s}}-\frac{R_{c}}{R_{s}}+\frac{D_{c}}{D_{s}}=\frac{D_{f}+D_{c}}{D_{s}}-\frac{R_{f}+R_{c}}{R_{s}}=1-1=0
$$

This means that the equation

$$
E_{f}=E_{c}
$$

always holds.

However, studies of the criterion have shown [3] that the optimum values obtained using this criterion depend on the initial composition of the material. Consequently, this criterion cannot be used to optimize separation processes, since it is not possible to determine whether the attainable efficacy is determined by the technical parameters of the process or differences in the composition of the starting material.

\section{OPTIMIZATION BY SEPARATION CURVES}

The second approach to the development of optimization methods is based on separation curves, which are invariant in relation to the initial composition [4-6]. Using these curves, it is possible to determine the entire set of optimal values of the technological parameters of the separation uniquely and with sufficient precision, within the limits of precision of measurement. However, methods of optimization based on separation curves suffer from a significant defect: they do not provide a quantitative characterization of efficacy.

The two approaches discussed above are mutually complementary. However, as they lead to different values of the optimum technological parameters [3], they cannot be used simultaneously. It would be useful, therefore, to develop a single method that would combine the advantages of both the optimization methods. We can accomplish this by taking the curves of fractional separation as a point of departure. The process of optimization can then be sought by minimizing the absolute degree of contamination of the two separation products.

Based on Fig. 1, this can be accomplished by minimizing one of the following expressions:

$$
E_{I}=R_{f}+D_{c}
$$


or, by maximizing the expression

$$
E_{I I}=D_{f}+R_{c}
$$

The optimization conditions for these relations, as is known, are

$$
\frac{d E_{I}}{d x}=0 ; \quad \frac{d E_{I I}}{d x}=0
$$

It can be shown that both of these relations give the same result:

$$
\frac{d E_{I}}{d x}=\frac{d D_{f}}{d x}+\frac{d R_{c}}{d x}=\frac{d \int_{0}^{x} q(x) d x}{d x}+\frac{d \int^{x_{\max }} n(x) d x}{d x}=0
$$

It is well-known that the derivative of an integral with variable upper limit and constant lower limit is equal to the expression under the integral sign at the point of the upper limit. Hence we obtain, from Eq. (7):

$$
\begin{aligned}
& q(x)-n(x)=0 \\
& \text { i.e. } q(x)=n(x)
\end{aligned}
$$

In view of Eq. (1), it is obvious that the particulate fraction will be divided into halves at the optimal point, i.e. the conditions of optimal efficacy are given by:

$$
F_{f}=F_{c}=0.5
$$

Only one point in Fig. 1 meets this condition, namely the point of intersection of curves $q(x)$ and $n(x)$ which lies on the ordinate of $x_{0}$. For any act of separation of a pourable material, it is, therefore, always possible to find a boundary of separation that corresponds to the optimum result, i.e. to find $x_{0}$ from the relation:

$$
F_{f}(x)=f(x)
$$

In the same way, using the separation curves it is possible to evaluate the optimum technological value of a parameter (e.g. stream velocity $w_{0}$ ) from the relation:

$$
F_{f}(x)=f(w)
$$

Let us now analyse Eqs. (5) and (6) for their correspondence to the boundary conditions of the separation:

1. In ideal separation, $D_{f}=D_{s}, R_{c}=R_{s}$ and $E_{I I}=1$, which reflects the meaning of the process.

2. In a simple division of the material into different products without change in granular composition

$$
D_{f}=\gamma_{f} D_{s} \quad \text { and } \quad R_{c}=\left(1-\gamma_{f}\right) R_{s}
$$




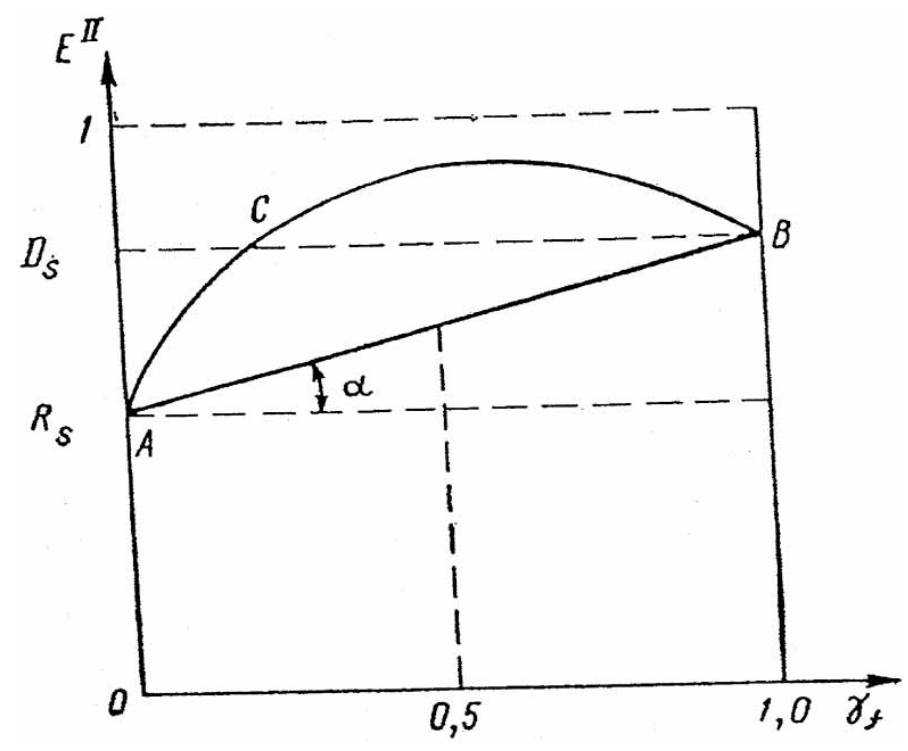

FIGURE 3 Graph of function $E_{I I}=f\left(\gamma_{f}\right)$.

In this case

$$
E_{I I}=\gamma_{f} D_{x}+R_{s}-\gamma_{f} R_{s}=R_{s}-\gamma_{f}\left(R_{s}-D_{s}\right)
$$

which, in the general case, is non-zero.

The above relation (10) is linear function:

$$
E_{I I}=f\left(\gamma_{f}\right)
$$

In the graph of this function (Fig. 3), point A corresponds to the value $\gamma_{f}=0$. From Eq. (10), $E_{I I}=R_{s}$ at that point. At point B the product assumes the value $\gamma_{f}=1$, while $E_{I I}=D_{s}$.

\section{RELATIONSHIP OF OPTIMIZATION METHODS}

In these coordinates, the straight line $\mathrm{AB}$ has a clear physical meaning: it is the line of zero efficacy according to the criterion $E_{I I}$, when the initial mixture is separated into any two portions without any change in granular composition in the separation products. When the separation products differ in granular composition, the curve defined by the relation $E_{I I}$ lies above the line $\mathrm{AB}$, assuming the position of the curve ACB. However, this relation is difficult to use in this form, because it acquires non-zero values in the absence of separation, leading to erroneous results. To overcome this difficulty, let us subtract the ordinate that defines zero efficacy at every point of the curve ACB:

$$
E_{I I I}=E_{I I}-\left[R_{s}-\gamma_{f}\left(R_{s}-D_{s}\right)\right]
$$


From this relation, and using Eq. (6), we obtain:

$$
E_{I I I}=D_{f}+R_{c}-R_{s}+\gamma_{f} R_{s}-\gamma_{f} D_{s}
$$

Recalling that $\gamma_{f}=\left(D_{f}+R_{f}\right)$ and carrying out the appropriate transformations, we find from Eq. (11):

$$
E_{I I I}=2\left(D_{f} R_{s}-R_{f} D_{s}\right)
$$

Let us analyse this relation.

1. For separation of the material into portions without change in granular composition:

$$
E_{I I I}=2\left(\gamma_{f} D_{s} R_{s}-\gamma_{f} D_{s} R_{s}\right)=0
$$

which corresponds to the physical meaning of the process.

2. For ideal separation:

$$
D_{f}=D_{s} \quad \text { and } \quad R_{f}=0, \quad \text { that is } E=2 D_{s} R_{s}
$$

This shows that the maximal attainable efficacy is dependent upon the initial granular composition of the material. This is an interesting result. However, it is more convenient to use Eq. (12) after normalizing it to ordinary percentage scale. We can write:

$$
E_{I V}=\frac{E_{I I I}}{2 R_{s} D_{s}}=\frac{2\left(D_{f} R_{s}-R_{f} D_{s}\right)}{2 R_{s} D_{s}}=\frac{D_{f}}{D_{s}}-\frac{R_{f}}{R_{s}}
$$

which is simply Hancock's formula for the efficacy of separation. Thus, analysis of the separation curves has led us to the well-known quantitative criterion.

This conclusion lays ground for a fundamentally new approach to the evaluation of the separation processes by combining positive features of both optimization approaches. The main transformations of expression $E_{I I}$ that lead us eventually to the relation for $E_{I V}$ have a clear graphical meaning. Reduction of $E_{I I}$ to zero-efficacy values is achieved by subtracting the distance from the $x$-axis to the line $\mathrm{AB}$ at every point, as is shown in Fig. 3. This is equivalent to shifting the point $\mathrm{A}$ to the origin of the coordinates and rotating the curve ACB by an angle:

$$
\alpha=\operatorname{arctg}\left(D_{s}-R_{s}\right)
$$

Reduction to unity by dividing by $2 D_{s} R_{s}$ does not affect the position of the optimum. The angle $\alpha$ varies between the limits, which are determined by the ratio of the fine and the coarse products in the starting mixture. For $D_{s}=1, \alpha=45^{\circ}$; for $R_{s}=1, \alpha=-45^{\circ}$, i.e. the interval of variation of this angle is given by:

$$
-45^{\circ} \leq \alpha \leq 45^{\circ}
$$


These operations make it possible to define the optimization parameters that will be invariant with respect to the composition of the mixture using the Hancock criterion, i.e. to combine the advantages of the two approaches in a single method.

\section{APPLICATION OF THE METHOD}

Let us demonstrate the method by a concrete example. A milled quartzite was separated using a classifier depicted in Fig. 4 [7,8]. The initial composition of the sample is given in Table I.

Figure 5 illustrates the dependence of the fine product on the air-flow velocity for a given composition. As is clearly seen from the graph, the yield of the fine product

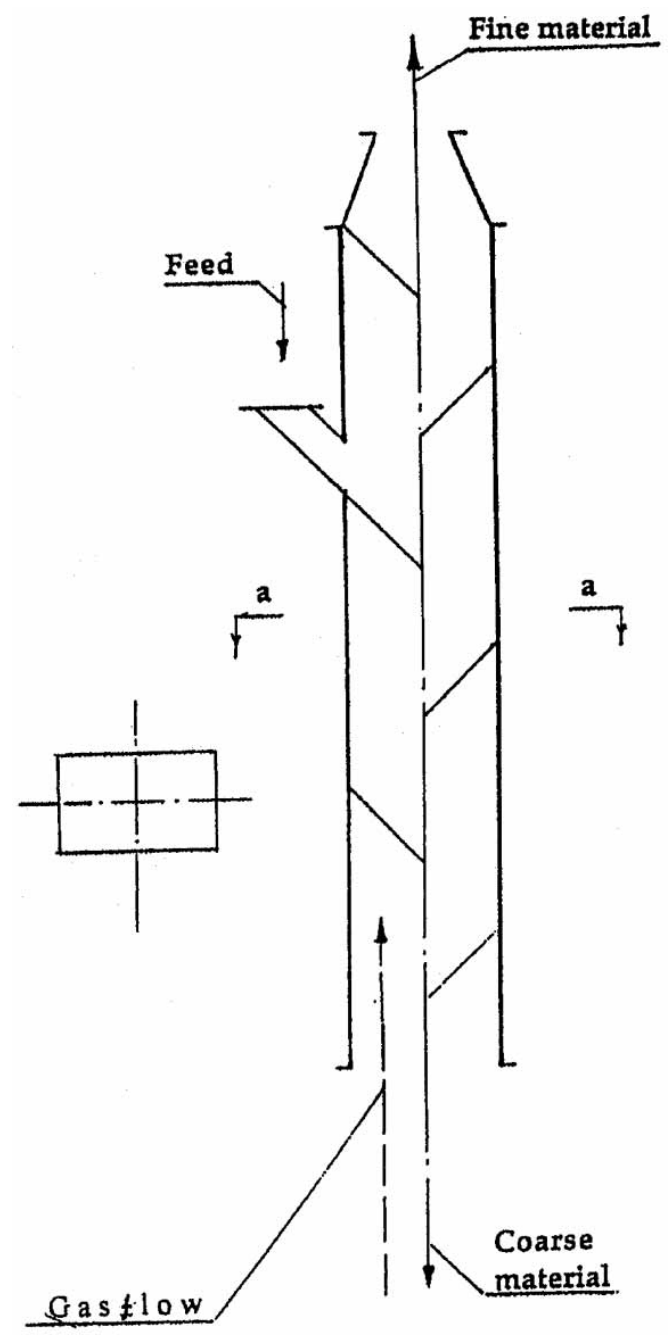

FIGURE 4 Casading classifier. 
TABLE I The grain-size distribution of milled quartzite

\begin{tabular}{lrccccrrr}
\hline $\begin{array}{l}\text { Screen opening } \\
\text { size } x(\mathrm{~mm})\end{array}$ & 7 & 5 & 3 & 2 & 1 & 0.5 & 0.25 & 0 \\
\hline Partial residue $r(\%)$ & 8.6 & 14.67 & 27.4 & 19.4 & 16.54 & 5.0 & 5.47 & 2.92 \\
Total residue $R_{s}(\%)$ & 8.6 & 23.27 & 50.67 & 70.07 & 86.61 & 91.61 & 97.08 & 100 \\
Total product $D_{s}(\%)$ & 91.4 & 76.73 & 49.33 & 29.93 & 13.39 & 8.39 & 2.92 & 0 \\
\hline
\end{tabular}

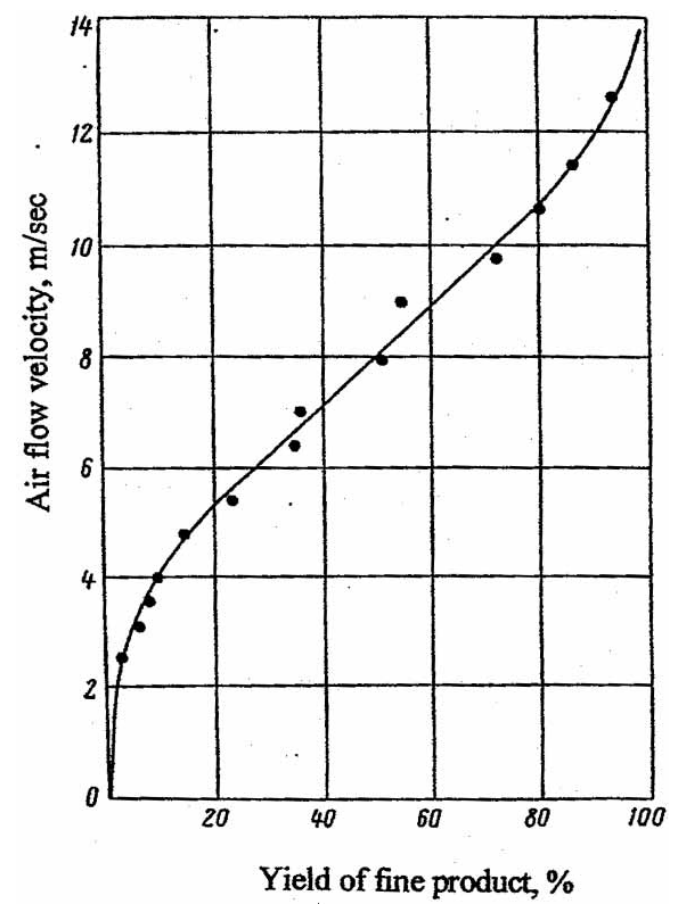

FIGURE 5 Yield of fine product as a function of air-flow velocity in the classification of milled quartzite.

uniquely relates the technological and design parameters of the process to the granular composition of the initial mixture.

Figure 6 shows the dependence of the Hancock criterion on the output of the fine product for different separation boundaries. Also shown are the directions of the axes for different boundary sizes for which the optimum output is being sought.

For comparison, the optimal values of the separation criteria, as determined by different approaches, are given in Table II.

We can note that the optimal efficacy, as determined using the separation curves, refers to the mid-point of the corresponding particulate-size interval.

To facilitate the comparison, the data given in Table II have been plotted in a graph, as shown in Fig. 7. The optimal yields, as determined from the separation curves, are designated by crosses. These values are clearly in good agreement with the optimal yields determined using $E_{I I}$; the scatter of points lies within the limits of accuracy of 


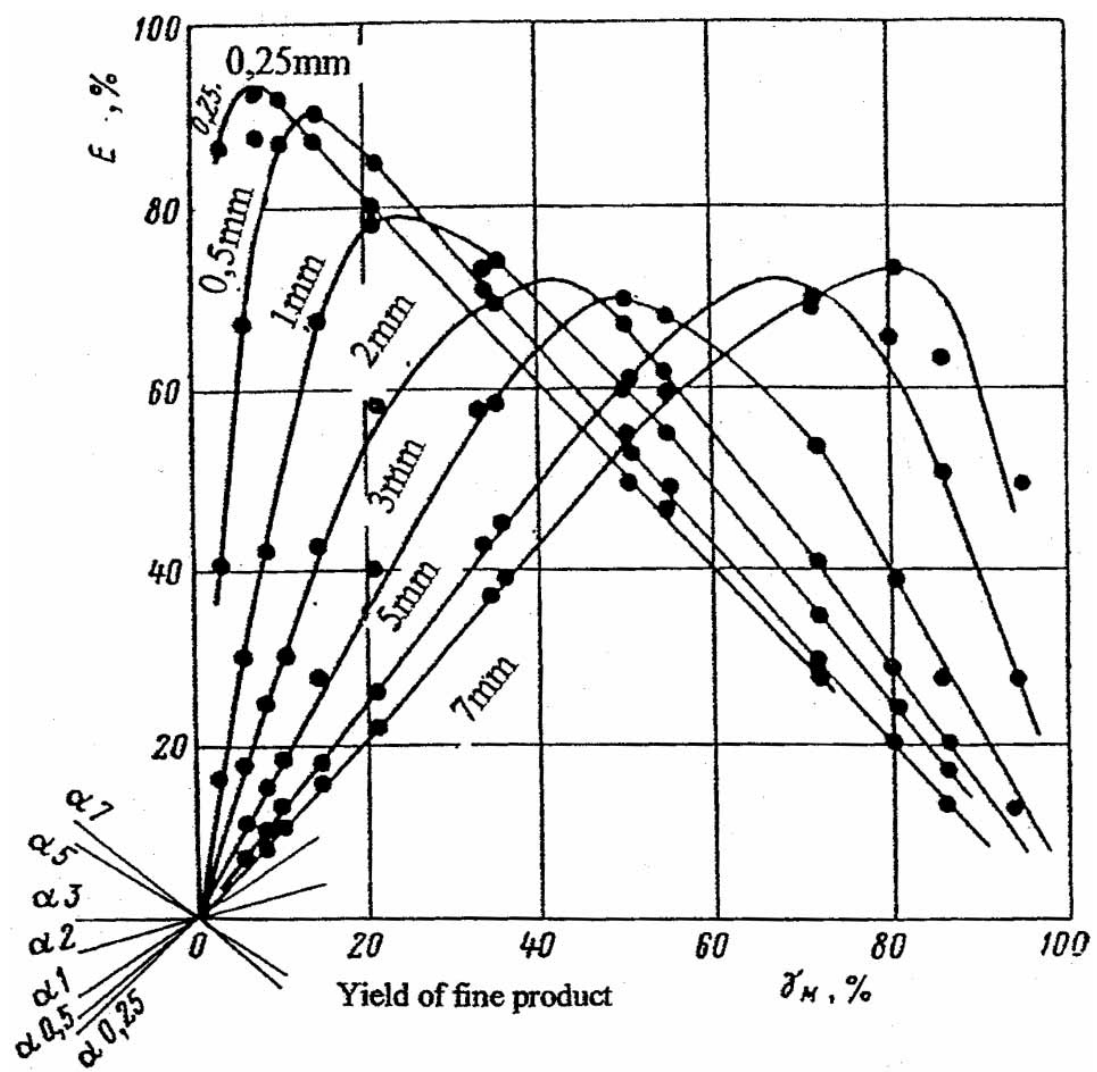

FIGURE 6 Dependence of Hancock's separation efficiency on the yield of fine product.

TABLE II Optimum separation indices derived by different methods

\begin{tabular}{lcccc}
\hline $\begin{array}{l}\text { Separation } \\
\text { boundary } \\
x(\text { mm })\end{array}$ & $\begin{array}{c}\text { Optimal yield } \\
\text { based on } \\
\text { Hancock, } E_{H}(\%)\end{array}$ & $\begin{array}{c}\text { Optimal yield } \\
\text { based on efficacy } \\
\text { criterion } E_{I I}(\%)\end{array}$ & $\begin{array}{c}\text { Optimal yield } \\
\text { based on separation } \\
\text { curves, } \gamma_{F}(\%)\end{array}$ \\
\hline 7 & 80 & 97 & 8.5 & - \\
5 & 67.5 & 82.5 & 6 & 85 \\
3 & 50 & 51 & 4 & 65 \\
2 & 42.5 & 30 & 2.5 & 45 \\
1 & 25 & 17.5 & 1.5 & 30 \\
0.5 & 15 & 11 & 16.5 & $(0.75)$ \\
0.25 & 7.5 & 6 & 5 & $(0.375)$ \\
\hline
\end{tabular}

Note: Values in parentheses indicate the mean size of a grain-size class $x$ in $\mathrm{mm}$.

the experiment. However, the relation determined by Hancock's method deviates significantly from the $E_{I I}$ curve, and the results given by different methods coincide only at a single point, namely for $D_{s}=R_{s}=50 \%$. Clearly, Hancock's method can be used objectively only in the latter situation. Under all other conditions, i.e. where $D_{s} \neq R_{s}$, optimization of separation processes may be carried out using the method proposed here. 


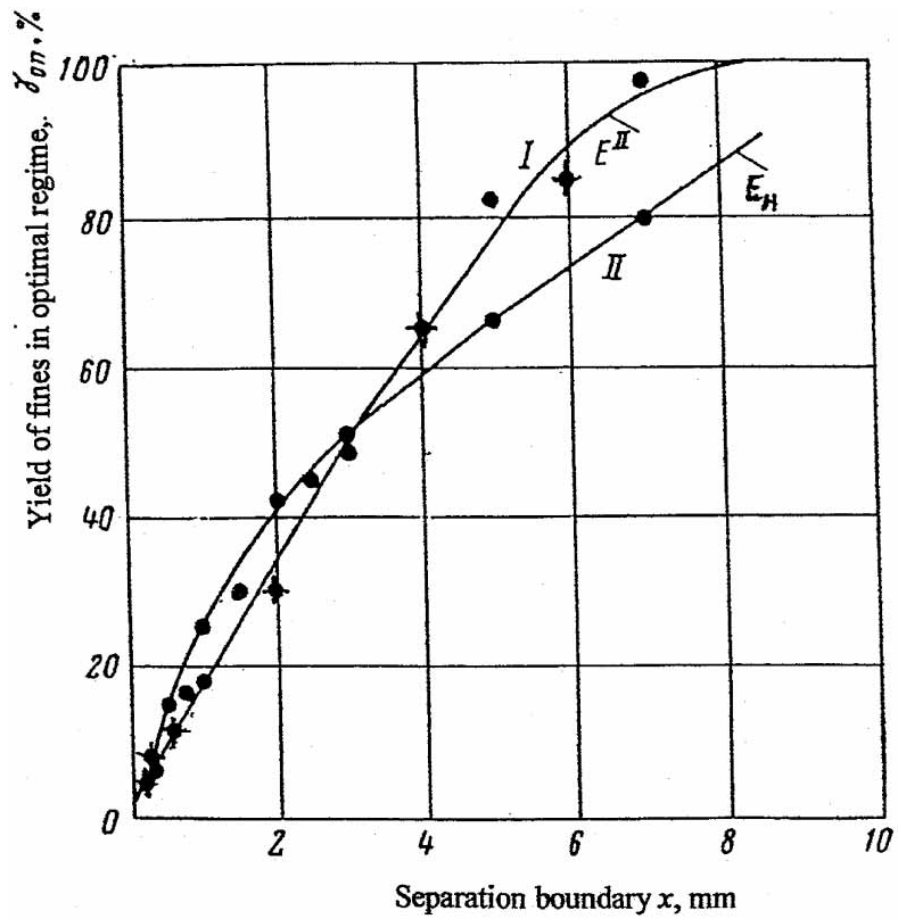

FIGURE 7 Correlation between different optimization methods depending on the separation boundary.

\section{CONCLUSION}

1. The Hancock criterion is applicable only in a particular case where $R_{s}=D_{s}$, and in all other cases it gives inaccurate results.

2. Under all other conditions, i.e. where $D_{s} \neq R_{s}$, the optimization of the separation processes can be carried out using a method proposed here.

\section{References}

[1] K.T. Hancock (1947). Calculating efficiencies from washery figures. Colliery Eng., May, June, July, 3.

[2] A.F. Taggart. Principles of Ore Beneficiation. Metallurgizdat, Moscow, 1958.

[3] M.D. Barsky. Classification of Powders. Nedra, Moscow (in Russian), 1980.

[4] K.F. Trompf (1937). Neue Wege fur die Beurteiling der Aufbereitung von Steinkohle. Gluckauf, 73.

[5] F.W. Mejer (1966). Die Trennsharfe von sichtern. Zement-Kalk-Gips, 6.

[6] F.G. Bond (1950). Calculated classifier data. Eng. and Min. Journ., 12.

[7] E. Barsky and M. Buikis (2004). Mathematical model for gravitational cascade separation of pourable materials at identical stages of a classifier. In: Progress in Industrial Mathematics, pp. 229-233, Springer Verlag, New York.

[8] M. Barsky and E. Barsky (2003). Correlation between particle spin and particle settling and the optimal stream velocity in gravity separation. Proc. XXII IMPC. Cape Town, South Africa, p. 378. 